\section{Nitrous oxide inhalation anaesthesia in the presence of intraocular gas can cause irreversible blindness}

\author{
A. J. Lockwood ${ }^{1}$ and Y. F. Yang ${ }^{2}$
}

IN BRIEF
- Nitrous oxide is a highly soluble rapidly
diffusing gas often used in sedation.
Ophthalmic patients sometimes have
intraocular gas injection as part of
their surgery.
- Exposure of nitrous oxide to patients
with intraocular gas can lead to an
elevated intraocular pressure and
blindness.

Nitrous oxide inhalation sedation is frequently used in dental and other surgical procedures. We report the case of a patient with intraocular gas who developed sudden elevation in intraocular pressure and blindness as a result of nitrous oxide anaesthesia. All medical and dental personnel administering nitrous oxide inhalation should be aware that this is contraindicated in patients with intraocular gas.

\section{INTRODUCTION}

Inhalation sedation with nitrous oxide and oxygen is commonly used as an alternative to general anaesthesia. The Poswillo Report ${ }^{1}$ has recommended its use in dentistry and it has been shown to result in less morbidity with shorter procedure time. ${ }^{2}$ An important contraindication to its use, however, is the presence of intraocular gas. Intraocular gases are frequently used in the surgical management of retinal diseases like retinal detachment. These gases may persist in the eye for up to three months following surgery. During this period, nitrous oxide inhalation whether used during a general anaesthesia or as inhalation sedation for dental procedures will cause rapid expansion of the intraocular gas bubble resulting in sight-threatening elevation of the intraocular pressure. ${ }^{3-10}$

${ }^{1 *}$ Specialist Registrar; ${ }^{2}$ Consultant Department of Ophthalmology, Queen Alexandra Hospital, Portsmouth, P06 3LY

*Correspondence to: Mr Alastair Lockwood

Email: alastairlockwood2000@yahoo.co.uk

\section{Refereed Paper}

Accepted 28 January 2008

DOI: $10.1038 /$ bdj.2008.158

${ }^{\oplus}$ British Dental Journal 2008; 204: 247-248

\section{CASE REPORT}

A 71-year-old man presented to his local ophthalmology unit with a two-month history of poor vision in the left eye. He was found to have a vitreous haemorrhage and subsequently developed a retinal detachment. He was referred to a vitreoretinal unit and underwent a vitrectomy procedure with retinal cryotherapy and a mixture of $86 \%$ purified air and 14\% perfluoropropane gas was injected into the vitreous cavity. On the first post-operative day, he could see hand movements with his left eye, the retina was attached and about $80 \%$ of his vitreous cavity volume was filled with the gas mixture. His intraocular pressure in the left eye was raised. Topical and systemic ocular antihypertensives were prescribed which reduced his intraocular pressure. He was discharged from inpatient care with the ocular antihypertensives. He was told that he had gas in his left eye and was advised to avoid air travel until the intraocular gas was completely absorbed.

He presented to his local casualty department the following evening with acute urinary retention. He was catheterised and admitted to a urological ward. Following an unsuccessful trial without a catheter, a transurethral resection of his prostate gland was arranged. Before operation he was reviewed at the local ophthalmology unit and was found to

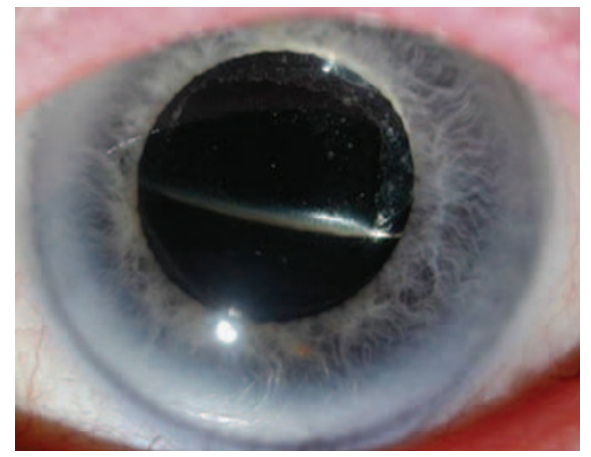

Fig. 1 An eye with about 50\% gas fill in the vitreous cavity examined using a pen-torch

be able to see hand movements with the retina attached. The vitreous cavity had a $60 \%$ gas fill, and his intraocular pressure was raised at $40 \mathrm{mmHg}$. Another topical ocular antihypertensive drug was added. His recent eye surgery, but not the presence of intraocular gas, was noted in the anaesthetic assessment chart. He underwent surgery under general anaesthesia. Intravenous agents were used for induction and the anaesthesia was maintained with volatile agents (sevoflurane) with 50-60\% nitrous oxide in oxygen as an adjuvant. The procedure lasted two hours. The next day he reported that he had intense pain in his left eye while recovering from the anaesthetic and had complete loss of vision in his left eye. An urgent ophthalmological opinion was sought. He had no light perception in his left eye, the pupil was mid-dilated and 
non-reactive and the intraocular pressure was $31 \mathrm{mmHg}$.

The patient was reviewed at the vitreo-retinal unit a week after his prostate surgery. The vitreous cavity had a $50 \%$ gas fill, and the retinal vasculature was attenuated. Three months after the retinal surgery the vision remained at 'no light perception' and the optic nerve was pale.

\section{DISCUSSION}

Intraocular gases are commonly used in retinal surgery, like retinal detachment surgery as an intraocular tamponade to hold the retina in position while the adhesive chorioretinal scar develops. Various long acting inert gases, such as sulphur hexafluoride or perfluoropropane, can be used as intraocular tamponading agents. ${ }^{11}$

The intraocular gas in retinal surgery is injected into the vitreous cavity. The kinetics of intraocular gases are similar to those of gases in any enclosed body cavity. When a bubble of gas is injected into the vitreous cavity nitrogen in the bloodstream will enter the gas bubble, causing it to expand. The solubility of nitrous oxide is 34 times that of nitrogen. ${ }^{3}$ If nitrous oxide is present in the blood stream it will rapidly enter the bubble, causing expansion and a dramatic increase in intraocular pressure. ${ }^{4,5}$ Nitrous oxide also rapidly leaves the bloodstream and gas bubble once inhalation is terminated, restoring the intraocular pressure. ${ }^{5}$

In the patient described above, the nitrous oxide used for anaesthesia during the prostrate surgery passed from the bloodstream into the intraocular gas bubble causing a rapid expansion of the bubble. The expanding bubble displaced the iris-lens diaphragm anteriorly, closing the outflow channels for intraocular aqueous fluid. The expansion of the intraocular gas bubble and the closure of the outflow channels led to a sudden massive increase in intraocular pressure beyond the perfusion pressure of the central retinal artery $(60-70 \mathrm{mmHg})$. At this point blood flow to the optic nerve head and other intraocular structures, like the iris, ceased. The surgery lasted two hours after which the nitrous oxide inhalation was stopped. As the nitrous oxide re-entered the bloodstream from the gas bubble, the gas bubble contracted and the iris-lens diaphragm returned to its normal position. The intraocular pressure decreased and reperfusion of the central retinal artery occurred. Over the two hour duration of the surgery, irreparable ischaemic retinal damage ocurred. ${ }^{12}$ A non-mobile middilated pupil is often a sign of ischaemic iris damage.

Other reports exist of nitrous oxide anaesthesia being used in patients with intraocular gas. ${ }^{6-10}$ In one case the optic nerve was noted to be pale intraoperatively. ${ }^{6}$ The longest reported period between a retinal operation with intraocular gas injection and subsequent visual loss from nitrous oxide anaesthesia is six weeks. ${ }^{9}$ Transient central retinal artery occlusion and iris-lens diaphragm displacement have been reported after experimental rapid expansion of an intraocular gas bubble. ${ }^{13}$

In England about 8,000 retinal surgical procedures are performed per year. ${ }^{14}$ After such surgery intraocular gas, if used, can persist for up to three months (Fig. 1). During this period while the intraocular gas is present, if a patient requires anaesthesia, nitrous oxide must not be used. To avoid iatrogenic irreversible blindness, all medical or dental personnel administering nitrous oxide inhalation should be aware of the complications of nitrous oxide inhalation in the presence of intraocular gas or gases in other body cavities.

The authors wish to thank the British Medical Journal for permission to publish this case where it first appeared (Br Med J 2002; 325: 532-533).

1. Report of an expert working party. General anaesthesia, sedation and resuscitation in dentistry. Publication no PL/CDO. London: Department of Health, London, 1990.

2. Hill FJ, Shephard A R. Orthodontic extractions: a comparative sturdy of inhalation sedation and general anaesthesia. Br Dent J 2000; 188: 329-331.

3. Wolf G L, Capuano C, Hartung J. Nitrous Oxide increases intraocular pressure after intravitreal sulfur hexafluoride injection. Anesthesiology 1983; 59: 547-548.

4. Smith R B, Carl B, Linn J G Jr, Nemoto E. Effect of nitrous oxide on air in vitreous. Am J Ophthalmol 1974; 78: 314-317.

5. Stinson T W III, Donion J V Jr. Interaction of intraocular air and sulfur hexafluoride with nitrous oxide: a computer simulation. Anesthesiology 1982; 56: 385.

6. Fuller D, Lewis M L. Nitrous oxide anesthesia with gas in the vitreous cavity (letter). Am J Ophthalmol 1975: 80: 778-779.

7. Smith B R. Nitrous oxide anesthesia with gas in the vitreous cavity (reply). Am J Ophthalmol 1975; 80: 778-779.

8. Yang $Y F$, Herbert $L$, Rüschen $H$, Cooling $R J$ J. Lesson of the week: Nitrous oxide anaesthesia in the presence of intraocular gas can cause irreversible blindness. Br Med J 2002; 325: 532-533.

9. Seaberg R R, Freeman W R, Goldbaum M H, Manecke G R Jr. Permanent postoperative vision loss associated with expansion of intraocular gas in the presence of a nitrous oxide-containing anesthetic. Anesthesiology 2002; 97: 1309-1310.

10. Lee $E J$. Use of nitrous oxide causing severe visual loss 37 days after retinal surgery. Br J Anaesth 2004; 93: 464-466.

11. Vygantas C M, Meymen G A, Daily M J, Ericson ES . Octafluorocyclobutane and other gases for vitreous replacement. Arch Ophthalmol 1973; 90: 235-236.

12. Hayreh S S, Weingeist T A. Experimental occlusion of the central artery of the retina. IV: Retinal tolerance time to acute ischaemia. Br J Ophthalmol 1980; 64: 818-825.

13. Dieker J P, O'Connor PS, Schacklett D E et al. Air travel and intraocular gas. Ophthalmology 1986; 84: 448-449.

14. Kilmartin D J, Dick A D, Forrester J V. Sympathetic ophthalmia risk following vitrectomy: should we counsel patients? Br J Ophthalmol 2000; 84: 448-449. 